\title{
Evaluation Method of Chemical Technology Safety Practical Operation Ability Based on Stochastic Model
}

\author{
Xiaotong Wang (iD \\ School of Safety Engineering, Shenyang Aerospace University, Shenyang 110000, China \\ Correspondence should be addressed to Xiaotong Wang; lgxr381@163.com
}

Received 18 September 2021; Revised 28 October 2021; Accepted 1 November 2021; Published 26 November 2021

Academic Editor: Deepak Gupta

Copyright (c) 2021 Xiaotong Wang. This is an open access article distributed under the Creative Commons Attribution License, which permits unrestricted use, distribution, and reproduction in any medium, provided the original work is properly cited.

\begin{abstract}
The current evaluation method does not consider the coordination and overall situation of various practical skills, which affects the evaluation contact degree and leads to hidden dangers of production safety. Therefore, an evaluation method of chemical technology safety practical operation ability based on a stochastic model is proposed. Using association rules to design the feature mining algorithm of practical operation ability, combined with crosslayer coding strategy, the mapping relationship between safety accidents and practical operation ability is established. The chemical safety practical operation skills are simulated by a random model, and the practical operation skills are classified and coordinated according to the simulation predictive control results. According to the coordination results, the evaluation index system is constructed, the index weight is determined, and the evaluation model of chemical technology safety operation ability is constructed. The experimental results show that the maximum connection degree of the evaluation method designed in this paper is $88.06 \%$, and the evaluation results are more accurate, which is helpful to improve the production safety of chemical enterprises.
\end{abstract}

\section{Introduction}

Security issues are not only related to production but also related to the overall situation of China's economic development, political stability, social harmony, and people's happiness [1]. As a pillar industry of China's economic development, the chemical industry is also a high-risk industry with many accidents due to its complex production process, various equipment and instruments, large-scale equipment, raw materials, and products, and most of them are dangerous goods. At present, the situation of chemical safety production in China is grim. All kinds of accidents not only directly cause casualties and economic losses but also bring a great burden to society, enterprises, and countless families. It not only affects the stability and unity within the society but also seriously affects China's good market investment environment and international image. The emergence of new technologies, new processes, and new products not only meets the growing needs of people but also increases the risk factors and safety risks in the production and operation process of enterprises. The inherent characteristics of chemical production, such as complex process, diverse raw materials, numerous equipment, fine operation, many hazardous chemicals, and dense distribution, have brought objective difficulties in risk management and control. Once an accident occurs, it is very easy to trigger a chain reaction, expand the harm of the accident, and even develop into a major safety accident, seriously threatening the personal safety of enterprise employees and residents near the plant [2]. It is an objective problem that chemical enterprise personnel must face to find and eliminate the potential safety hazard and accident risk in the process of production and operation.

Reference [3] proposed the research on the safety risk status index system of chemical enterprises based on analytic hierarchy process and investigated the safety risk status of chemical enterprises from the aspects of personnel technical level, equipment failure, major hazard sources, production process, environment, accidents, certification, safety assessment, and emergency response. The safety risk state index system of chemical enterprises is constructed from six aspects: personnel, equipment, materials, methods, environment, and safety management, and the key index system of safety risk state early warning is put forward. The analytic 
hierarchy process is used to determine the index weight of safety risk status of chemical enterprises and finally determine the classification standard of safety risk status of chemical enterprises. Reference [4] proposed the research on safety early warning management of coal face based on expert system. Combined with the investigation of coal face in a large number of coal mines, a safety early warning expert system was constructed. This paper describes the classification, acquisition mechanism, and representation of knowledge and introduces the system structure, reasoning mechanism, and the establishment of knowledge base. In the process of construction operation in chemical enterprises, there are huge risks. Due to the complex procedures and various processes, it has a strong dependence on the safe operation behavior of workers. Once workers operate carelessly, it may cause serious accident consequences. It is of great practical significance to carry out the research on the evaluation of the practical operation ability of chemical technology safety to effectively improve the production safety of chemical enterprises. Nowadays and even in the future, the chemical industry will flourish. At present, China's relevant safety training, especially the practical training system, is not perfect, which leads to the lack of practical skills or unskilled chemical enterprise personnel, which easily produces unsafe behavior and leads to safety accidents. Therefore, it is necessary to evaluate the practical ability of chemical technical personnel, put forward the optimization angle of training system and specific measures to improve the practical ability, and apply it to practical training assessment and practice. The stochastic model can predict according to the current state, solve an open-loop optimization problem online according to the given objective function and constraints, and act the first element of the optimized control sequence on the controlled object. When it reaches a new state, it refreshes the optimization problem with the new measured value or estimated value and solves it repeatedly [5]. Therefore, the stochastic model can get good prediction optimization feedback results.

Therefore, this paper designs an evaluation method of chemical technology safety operation ability based on a random model. Through the correlation analysis between assessment items and safety accidents, this paper designs a feature mining algorithm of safety operation ability and obtains the correlation between data and safety accidents combined with crosslayer coding strategy. According to the random model, the construction safety operation skills are simulated, classified, and coordinated. When calculating the control strategy of a single safety operation skill, consider the behavior of other skills, so as to realize the global coordinated control of the whole operation ability and improve its connection degree. According to the coordination results, the evaluation index system is constructed, the evaluation index weight is determined, and the evaluation model of chemical technology safety operation ability is constructed [6-8], in order to continuously improve the practical training system, continuously improve workers' safety awareness and practical skills, reduce and control unsafe behaviors, and reduce the incidence of safety accidents.

\section{The Evaluation Method of Chemical Technology Safety Practical Operation Ability Based on Stochastic Model}

2.1. Design of Security Operation Ability Feature Mining Algorithm Based on Association Rules. In order to accurately evaluate the safety operation ability of technicians in chemical enterprises, firstly, the algorithm is used to mine the characteristics of operation ability. Through the correlation analysis between the assessment items and safety accidents, the importance of the assessment items and the correlation between the items are quantitatively evaluated, which provides valuable reference for the targeted training and assessment of practical skills. Association rule mining is one of the important branches of data mining, which is used to mine the correlation between valuable data items from a large number of data. The results of association rule mining mainly describe the association and degree between objects in the database or data warehouse [9]. Because the original data cannot directly reflect the correlation between chemical safety accidents and evaluation indexes, in order to better organize and summarize the correlation between existing capacity data and safety accidents, this paper proposes a crosslayer coding strategy. In this strategy, the original data is regarded as the bottom data of mining database, and the index item of practical ability is regarded as the middle layer. The original data is summarized to the corresponding middle layer through coding technology, and finally, the association rules between the target layer and the middle layer are found [10]. The flow chart of association rule mining algorithm is shown in Figure 1.

According to the analysis of Figure 1, first, through crosslayer coding, the original data is mapped from the basic item set to the interesting class item set in the form of coding. Class item sets are summarized from the original data and use the event sets of interest to objects. The binary sequence formed by the mapping from class to evaluation target is called basic antigen. In the process of class item mapping, the matching degree between basic item set index items and class item set index items is expressed by weight value, which is called mapping weight value [11]. For example, a "judgment error" in the basic item set and the corresponding index items in the category item set include "communication and communication ability," "decisionmaking ability," "stress and fatigue," and "situational awareness and risk assessment ability." Basic antigen and mapping weight value reflect the numerical relationship between basic item and category index. It can be expressed as

$$
A_{i}=\left[W_{i}^{T} B_{i}\right]^{T} .
$$

In formula (1), $A_{i}$ represents the weighted value of the mapping; $i$ represents the number of class items; $T$ represents matrix transpose; $W_{i}$ represents the mapping weight value; and $B_{i}$ represents the basic antigen sequence of the class. Then, the numerical calculation formula of any class term is as follows: 


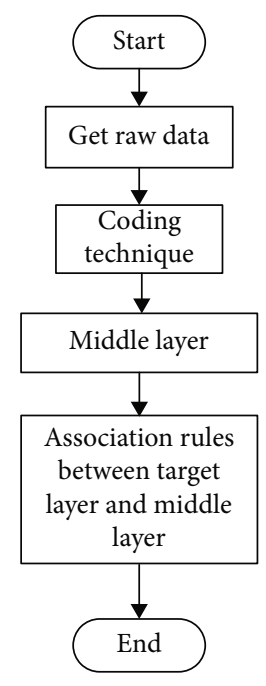

FIGURE 1: Algorithm flow of association rule mining.

$$
z_{i}=\frac{A_{i} \beta}{\left(\sum_{1}^{k} c \alpha\right)_{\max }}
$$

In formula (2), $z_{i}$ represents the class value; $\beta$ represents the number of bits in the binary representation; $k$ represents the number of basic items contained in the largest class item; $c$ represents the weight value of nonzero mapping; and $\alpha$ is the basic item. Then, we can mine the association rules of the coded class item set to find out the potential relationship between the interest index and the mining target [12]. Selecting the appropriate fitness function is a key problem of association rule mining algorithm, and it is also the key to the convergence speed of the algorithm [13]. The fitness function expression is as follows:

$$
g\left(z_{i}\right)=\lambda_{1} \frac{u\left(z_{i}\right)}{u_{\min }}+\lambda_{2} \frac{d\left(z_{i}\right)}{d_{\min }} .
$$

In formula (3), $g$ represents the fitness value of the class item; $\lambda_{1}, \lambda_{2}$ are adaptation coefficients, the sum of which is $1 ; u$ represents support; $u_{\min }$ represents the support threshold; $d$ represents the confidence level; and $d_{\text {min }}$ represents the confidence threshold. The above process establishes the mapping relationship between safety accidents and practical operation ability index, forms the data mining chain of this paper, and provides the basis for the evaluation of safety practical operation ability.

2.2. Coordination of Chemical Safety Practice Skills Based on Stochastic Model. Chemical safety accidents and disasters often occur suddenly, which could not be predicted before. In order to reduce the accident harm to the greatest extent, the most important thing is to do a good job with safety operations. Safety operation work runs through the whole chemical enterprise workflow. When there is no accident, it is necessary to regularly monitor the storage points of dangerous chemicals and storage tanks of inflammables and explosives in the chemical industry park or enterprise production plant and take corresponding measures to eliminate risk factors and potential hazards, so as to prevent accidents. After the accident, it is necessary to know the situation of the accident, confirm the type of the accident, adjust the emergency plan according to the characteristics of the accident and the actual situation of the plant, carry out rescue, control the development trend of the accident, prevent the expansion of the harm of the accident and the occurrence of derivative disasters, and rescue the trapped personnel in time. After the accident is effectively controlled, it is necessary to help the enterprise to resume production as soon as possible, carry out strict safety hazard investigation in the plant, summarize the specific situation of the accident and the emergency rescue process, and timely repair the emergency plan and hazard protection measures for the existing problems. The evaluation of practical operation ability is based on state feedback, so it is only applicable to the situation of measurable state. However, due to the existence of uncertain factors in the operational capability, the state cannot be accurately estimated. In many cases, there may be conflicts before the control objectives of practical ability, leading to unsafe behavior. When there is a certain degree of coordination between the practical skills, it can effectively avoid unsafe behavior and improve the comprehensive practical ability. The random model is a group of random samples randomly selected everywhere, so the treatment effect is random and varies with different experiments. If the experiment is repeated, a new set of samples must be randomly selected from the population. The introduction of the random model can make individual observations have a certain correlation, improve the coordination between practical skills, and get good feedback results. According to the stochastic model, the chemical safety practical skills are simulated, and the practical skills are classified and coordinated according to the model predictive control results [14]. By considering the behavior of other skills when calculating the control strategy of a single safety practical skill, the global coordinated control of the whole practical ability is realized. For this reason, the feasible candidate solution of practical ability can be expressed as follows:

$$
p=[v[n-1] \cdots v[n+m-1] 0]^{T} .
$$

In formula (4), $p$ represents the augmented vector, that is, the feasible candidate solution; $v$ represents the perturbation sequence of a single practical skill; $n$ represents the number of skills that need to be coordinated; and $m$ represents the number of skills that do not need to be coordinated [15]. In order to achieve the optimal coordination of operational capability, the weighted sum of local control functions is defined as follows:

$$
f(v)=\min \theta_{1} p+\sum_{1}^{n} \theta_{2} l
$$

In formula (5), $f(v)$ is the weighted sum of local control functions; $\theta_{1}$ is weight matrix; $\theta_{2}$ represents weighted cost; and $l$ is the limit value of infinite time-domain control 
function [16]. It can be seen that in order to promote the coordination among practical skills, the stochastic model designs a perturbation sequence for each coordination subskill [17]. At the same time, the weighted cost of each practical skill is considered in the local control function of comprehensive practical ability. Therefore, the comprehensive practical ability can predict and improve the comprehensive level of the whole practical ability by sacrificing the local ability of each subskill [18]. In order to optimize the output of the stochastic model, it is necessary to deal with the probability constraints. For any perturbed sequence, the following iterative feasible constraints need to be satisfied:

$$
h(v)=p t(v)+e(v)
$$

In formula (6), $h(v)$ represents the feasible constraint matrix of iteration; $t(v)$ is the displacement matrix; and $e(v)$ represents the maximum element value of the matrix. In order to ensure the replaceability of the worst-case value, the perturbation sequence is decomposed to ensure that the probability constraint is satisfied, and under this constraint, the optimization result of iterative feasibility is obtained [19]. The above process can realize the simulation results of comprehensive practical ability under coupling constraints and realize the coordination among practical skills. The coupling probability constraints among multiple practical skills are transformed into deterministic local constraints; thus, the predictive control of the stochastic model is transformed into quadratic programming solution [20]. This method can ensure the iterative feasibility of the optimization problem for local constraints and coupling constraints and make the evaluation of practical operation ability more coordinated and global.

2.3. Establish the Evaluation Index System of Practical Ability. According to the coordination results, the evaluation index system is constructed and the weight of evaluation index is determined. According to the task and content of comprehensive practical skills, the first level evaluation index is divided into four types: prevention, preparation, response, and recovery [21]. In prevention ability, fully learn the professional knowledge and technology of hazardous chemicals and safety emergency, and improve their own quality and ability. Make clear all kinds of reactions, operation procedures, and safety protection measures in the production process of chemical industry park or enterprise, and understand the types, hazards, protection measures, and distribution of all kinds of hazard sources in the plant. Carry out regular inspection on the plant area, eliminate risk factors and potential safety hazards of accidents, and formulate emergency plans and protective measures for possible accidents [22]. In preparation ability, prepare for possible accidents or equipment containers with high risk coefficient, such as the establishment of emergency rescue command center, reasonable planning and design of internal emergency escape routes, and the development of risk management system. Only with the ability of preparation can we not panic; follow blindly, calmly, timely, and quickly; effec- tively carry out emergency rescue; and ensure the safety of personnel [23]. In response ability, take measures immediately before the accident does not cause particularly serious harm to prevent the expansion of the scope and harm of the accident and effectively guarantee the safety of personnel and property [24], including accident and disaster identification, emergency rescue command and decision-making, and effective use of emergency equipment and materials. In recovery ability, after the accident is effectively controlled, it is necessary to carry out strict and comprehensive hidden danger investigation in the plant and help the enterprise to deal with the aftermath and recover, including potential safety hazard investigation, accident and disaster investigation and analysis, timely revision of emergency plan, strengthening enterprise accident protection measures, and resuming production $[25,26]$. The above four first-level indicators are set as second-level indicators according to the specific situation to form the evaluation index system. After the evaluation index system is constructed, the index weight is further determined. When calculating the weight, it needs to meet the basic consistency and order consistency. That is, in an evaluation index system, if the relative importance of index $x$ is twice of index $y$ and the relative importance of index $y$ is 3 times of index $z$, the relative importance of index $x$ is 6 times of index $z$. At the same time, the weight coefficient of the evaluation index is determined. The calculation formula is

$$
\omega_{x}=\left(1+\sum_{2}^{x} \prod_{y}^{x} \gamma_{y}\right)^{-1}
$$

In formula (7), $\omega_{x}$ represents the weight of evaluation index; $x, y$ represent any two evaluation indexes; and $\gamma_{y}$ is the scale of relative importance among evaluation indexes. Based on the above evaluation index system and the weight coefficient, the evaluation model of chemical technology safety operation ability is further constructed.

2.4. Construction of Evaluation Model for Practical Operation Ability of Chemical Technology Safety. In the process of practical operation ability evaluation, there are often some situations that lack enough data or some indexes are difficult to quantify. It is difficult to use a quantitative index to express the evaluation results. At this time, the fuzzy evaluation method can be used to convert some qualitative indexes into quantitative indexes according to the membership theory in fuzzy mathematics thinking, which means that the concept of fuzzy mathematics can be used to make a general evaluation of the objects or objects affected by many factors. Therefore, this paper uses the concept of fuzzy mathematics to build a fuzzy rating model for the evaluation of safety practical operation capability and evaluates the safety practical operation ability of chemical technology. Because of the many factors, it involves a wide range of aspects, and the degree of influence in various aspects is different. When using the first-level model, it may be ignored because the weight of the one hand is small, so the results are not so accurate. Therefore, this paper uses a multilevel 
TABLE 1: Evaluation index and its weight.

\begin{tabular}{|c|c|c|c|}
\hline First-level indicators & Weight & Second level indicators & Weight \\
\hline \multirow{3}{*}{ Preventive capacity } & \multirow{3}{*}{0.415} & Monitoring and early warning & 0.326 \\
\hline & & Protective measures & 0.458 \\
\hline & & Operation process & 0.216 \\
\hline \multirow{3}{*}{ Preparation ability } & \multirow{3}{*}{0.115} & Use of emergency equipment & 0.418 \\
\hline & & Hidden danger investigation and control & 0.201 \\
\hline & & Emergency avoidance & 0.381 \\
\hline \multirow{3}{*}{ Responsiveness } & \multirow{3}{*}{0.323} & Accident identification and control & 0.354 \\
\hline & & Signal communication & 0.286 \\
\hline & & Rescue treatment & 0.360 \\
\hline \multirow{3}{*}{ Resilience } & \multirow{3}{*}{0.147} & Accident summary and analysis & 0.392 \\
\hline & & Dealing with the aftermath & 0.133 \\
\hline & & Restoration and reconstruction & 0.475 \\
\hline
\end{tabular}

model to evaluate fuzzy decisions. All factors are divided into several subsets according to the fields involved. Each subset is evaluated according to the first-order model first, and then, a corresponding number of first-order fuzzy evaluation vectors will be obtained. If each subset is regarded as an element, the first-order fuzzy evaluation vector can be evaluated as a single factor. By calculating the distributable weight, the multilevel fuzzy evaluation vector is obtained. The evaluation set is a set of evaluation results for the evaluation object, which is generally a degree description or a numerical interval. The number of comments in common evaluation sets is usually odd. The selected comment set in this paper is \{very good, good, general, bad, very bad\}. In the use of fuzzy evaluation method, the proper fuzzy operator should be selected for matrix composite calculation. In this paper, we choose weighted average operators, and by assigning values, all cases are considered according to weight, so as to avoid omission. In the process of fuzzy evaluation, membership function is also very important, because it can have a direct impact on the results. The expression of membership function is different in different situations, and its creation method is not fixed, so it needs to be modified constantly according to the actual situation. According to the steps of multilevel fuzzy evaluation, a fuzzy evaluation vector can be obtained. The evaluation index is processed by a weighted average principle. The five evaluation grades in the evaluation set are assigned. The final evaluation results can be expressed as follows:

$$
F=\frac{\sum_{i=1}^{s} \phi_{i} p_{i}}{\sum_{i=1}^{s} \phi_{i}}
$$

In formula (8), $F$ represents the evaluation result; $s$ is the number of evaluation vectors; $i$ is the number of vector elements; $\phi_{i}$ represents fuzzy evaluation vector; and $p_{i}$ is the evaluation level assignment. The evaluation model is as follows: (1) factor set: according to the evaluation index system, the fuzzy evaluation factor set is established; (2) comment set: it is divided into five levels to evaluate the practical ability; (3) index weight set: the weight coefficient of the determined evaluation index is used as the weight set of the evaluation; (4) membership degree: because most of the indexes in the evaluation index system of safety production emergency preparedness ability established in this paper are fuzzy, fuzzy judgment method is used to calculate the membership degree of the indexes; (5) operation model: the weighted average operator is used to fully consider the high weight index and low weight index; and (6) evaluation result processing: the final evaluation result is calculated by a weighted average method. Based on the above process, the evaluation method of chemical technology safety operation ability based on the stochastic model is designed.

\section{Experiment}

3.1. Experimental Preparation. In order to test the effectiveness of the evaluation method designed in this paper, the following experimental test was performed. Taking a chemical enterprise as the evaluation object, the safety operation ability was evaluated and analyzed. According to the evaluation method designed in this paper, the evaluation system is established and the weight is assigned. The specific results are shown in Table 1.

According to the established evaluation index system, the evaluation model is constructed. The safety data of technicians in chemical enterprises are input into the evaluation model to complete the test of the evaluation method.

3.2. Experimental Results and Analysis. The hardware of the test platform in the experiment is mainly composed of CPU, hard disk, etc. The framework is TensorFlow, and the software of the test platform is mainly composed of the kernel zImage and U-Boot.bin. The number of cores of the CPU is eight cores; the processor is AMD and has 16 threads. The hard disk has a capacity of $128 \mathrm{~GB}$ and uses a SATA interface.

Taking the safety practical operation ability evaluation method based on random model design as the experimental group and selecting the practical operation ability evaluation method based on AHP and expert survey method as the control group, the comparative experiment was carried out. 


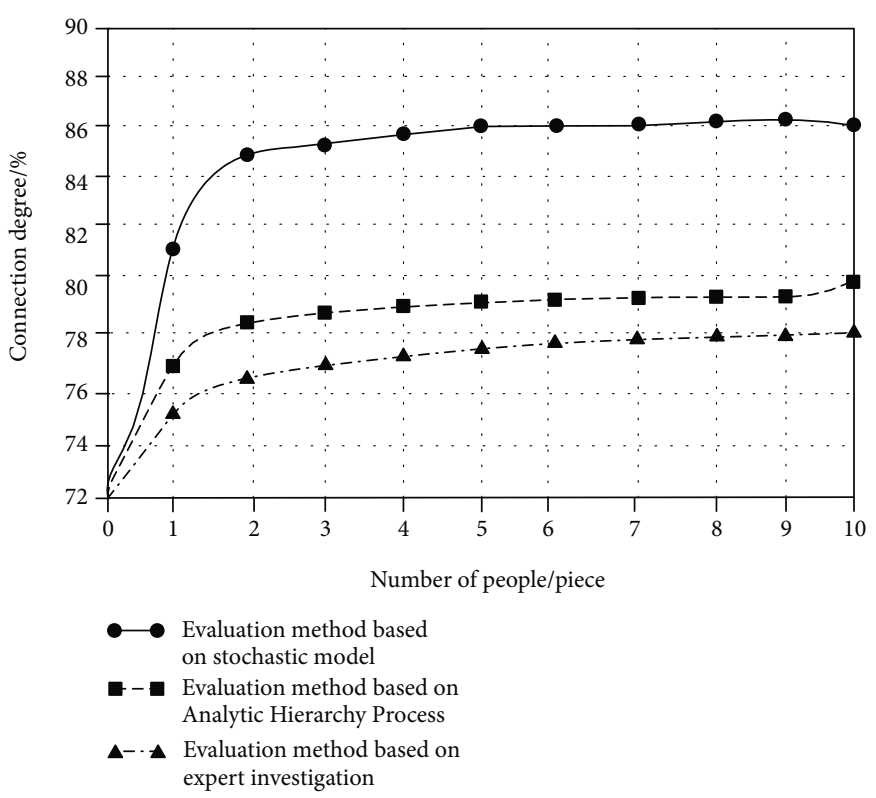

(a) Technical director

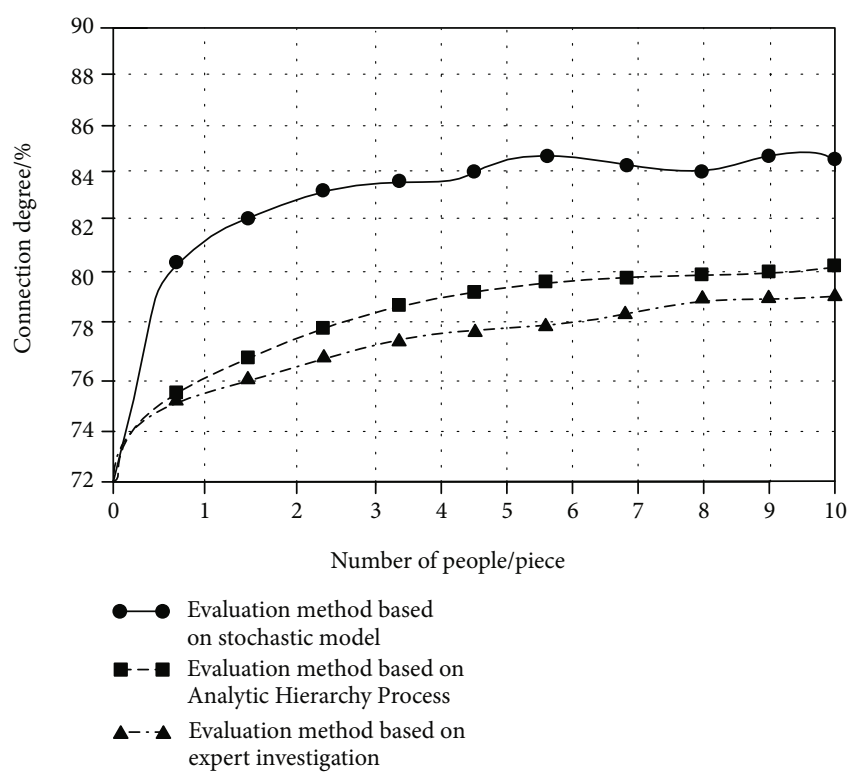

(b) Equipment technician

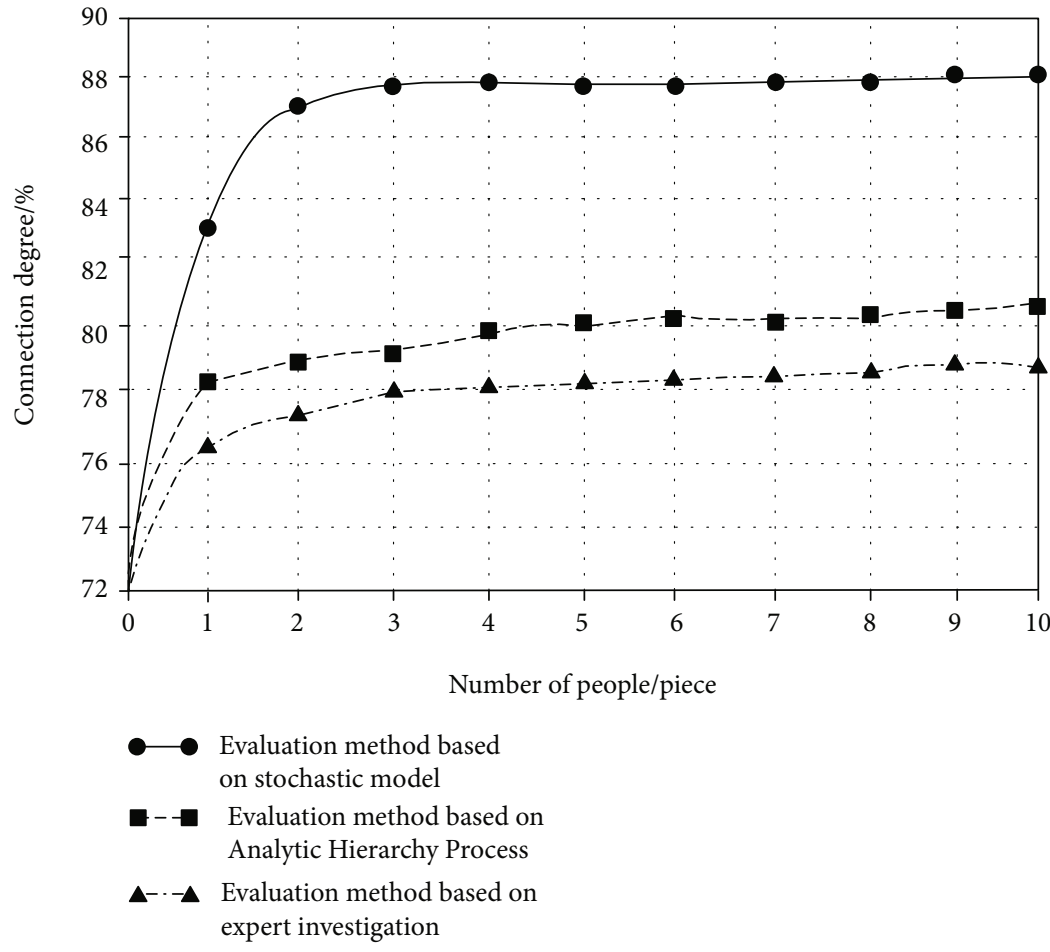

(c) Safety officer

FIgURE 2: Evaluation results of practical ability.

In order to compare the evaluation accuracy of different methods, the value of connection degree is used to measure the correlation between evaluation objectives and evaluation grades. The calculation formula of the connection degree is as follows:

$$
D=\eta_{1} a+\eta_{2} b+\eta_{3} c
$$

In formula (9), $D$ represents the connection degree of evaluation items; $a$ is weighted degree; $b$ is difference degree; $c$ is opposite degree; and $\eta_{1}, \eta_{2}, \eta_{3}$ represent the weight of secondary indicators. According to the above formula, the connection degree of evaluation target under different grades can be obtained. The larger the connection degree is, the more consistent the evaluation target is with the evaluation grade. Therefore, the greater the degree of connection, the more accurate the results of the method. In order to verify the feasibility, according to the determined index system, the results of the technical director, equipment technician, and safety officer are selected as the samples, and the 
practical ability of the experimental group and the control group is evaluated by three methods. The results are shown in Figure 2.

According to the evaluation results in Figure 2, although the technical director, equipment technician, and safety officer have the same assessment hierarchy, due to their different management level and operation level, the evaluation results obtained by using the three evaluation methods are different. Using the evaluation method of this paper to evaluate the technical director, equipment technician, and safety officer, the contact degree is $86.13 \%, 84.22 \%$, and $88.06 \%$, respectively. Based on the analytic hierarchy process, the technical director, equipment technician, and safety officer were evaluated, and the contact degrees were $79.82 \%$, $80.26 \%$, and $80.46 \%$, respectively. Based on the expert investigation method, the technical director, equipment technician, and safety officer were evaluated, and the contact degrees were $77.93 \%, 79.17 \%$, and $78.68 \%$, respectively. Compared with the evaluation results based on AHP and expert survey, the connection degree of the evaluation results based on the random model is higher, so the evaluation objective and evaluation grade of the design method in this paper are more relevant, and the evaluation results are more accurate. The evaluation method designed in this paper can realize the evaluation of practical ability of different chemical workers, help to train chemical technicians, and improve the production safety of chemical enterprises.

\section{Conclusion}

The evaluation method of safety practical operation ability proposed in this paper can promote the improvement of practical operation training to a certain extent, which is conducive to the reform and innovation development of chemical enterprises, enhance the safety behavior of chemical technicians, and promote the prevention and safety control of operation accidents. With the rapid development of modern science and technology, it is necessary to establish an evaluation network technology platform with the combination of big data analysis and other modern scientific and technological achievements. And the real-time evaluation and dynamic analysis of the practical operation level of chemical technical personnel are carried out. In view of the problems in each stage, we can analyze the reasons in time and put forward the optimization path, so as to promote the practical ability of chemical technicians. The analysis of the results shows that the connection degrees obtained by the proposed method are $86.13 \%, 84.22 \%$, and $88.06 \%$, respectively. The connection degrees of the evaluation results are higher. The designed evaluation method can realize the evaluation of the practical operation ability of different chemical personnel, help to train chemical technicians, and improve the production safety of chemical enterprises.

Due to the complex factors affecting the safety practical operation ability of chemical technology, there may be some limitations. In terms of screening all factors that may affect the effectiveness of practical operation examination, more comprehensive observation and further in-depth research are needed in the future.

\section{Data Availability}

The datasets used and/or analyzed during the current study are available from the corresponding author on reasonable request.

\section{Conflicts of Interest}

It is declared by the author that this article is free of conflict of interest.

\section{References}

[1] G. C. Ta, M. Sultan, K. E. Lee et al., "A proposed integrated framework for chemical safety and chemical security," Journal of Chemical Education, vol. 97, no. 7, pp. 1769-1774, 2020.

[2] S. Chen and S. Hu, "Safety integrity assessment and verification of typical chemical plant," Modern Chemical Industry, vol. 40, no. 5, pp. 10-13, 2020.

[3] F. Wu, L. Cheng, Y. Yu, and J. Sun, "Research on the index system of chemical enterprise safety risk state based on analytic hierarchy," E3S Web of Conferences, vol. 245, no. 6, p. 03082, 2021.

[4] F. Meng, Y. Fang, and C. Li, "Research on safety early warning management of coal mining face based on expert system," IOP Conference Series Materials Science and Engineering, vol. 490, no. 6, article 062056, 2019.

[5] S. Golshan, R. Zarghami, and N. Mostoufi, "A hybrid deterministic-stochastic model for spouted beds," Particuology, vol. 42, no. 1, pp. 104-113, 2019.

[6] Z. Niu, B. Zhang, D. T. Li et al., "A mechanical reliability study of 3-dB waveguide hybrid couplers in submillimeter and terahertz bands," Frontiers of Information Technology \& Electronic Engineering, vol. 22, no. 8, pp. 1104-1113, 2021.

[7] B. Li, G. Xiao, R. Lu, R. Deng, and H. Bao, "On feasibility and limitations of detecting false data injection attacks on power grid state estimation using D-FACTS devices," IEEE Transactions on Industrial Informatics, vol. 16, no. 2, pp. 854-864, 2020.

[8] Z. Lv, D. Chen, R. Lou, and H. Song, "Industrial security solution for virtual reality," IEEE Internet of Things Journal, vol. 8, no. 8, pp. 6273-6281, 2021.

[9] F. Moslehi, A. Haeri, and F. Martínez-Lvarez, "A novel hybrid GA-PSO framework for mining quantitative association rules," Soft Computing, vol. 24, no. 6, pp. 4645-4666, 2020.

[10] B. Shazad, H. U. Khan, F. M. Zahoor-ur-Rehman et al., "Finding temporal influential users in social media using association rule learning," Intelligent Automation and Soft Computing, vol. 26, no. 1, pp. 87-98, 2020.

[11] G. Czibula, I. G. Czibula, D. L. Miholca, and L. M. Crivei, "A novel concurrent relational association rule mining approach," Expert Systems with Applications, vol. 125, no. JUL., pp. 142156, 2019.

[12] S. Nemet, D. Kukolj, G. Ostojic, S. Stankovski, and D. Jovanovi, "Aggregation framework for TSK fuzzy and association rules: interpretability improvement on a traffic accidents case," Applied Intelligence, vol. 49, no. 11, pp. 3909-3922, 2019. 
[13] P. D. Sheena Smart, K. K. Thanammal, and S. S. Sujatha, "A novel linear assorted classification method based association rule mining with spatial data," Sādhanā, vol. 46, no. 1, pp. 112,2021

[14] F. M. Ibanez, I. Idrisov, F. Martin, and A. Rujas, "Design balancing systems for supercapacitors based on their stochastic model," IEEE Transactions on Energy Conversion, vol. 35, no. 2, pp. 733-745, 2020.

[15] M. Lopez-Garcia, M. F. King, and C. J. Noakes, "A multicompartment sis stochastic model with zonal ventilation for the spread of nosocomial infections: detection, outbreak management, and infection control," Risk Analysis, vol. 39, no. 8, pp. 1825-1842, 2019.

[16] E. Ferrero and D. Oettl, "An evaluation of a Lagrangian stochastic model for the assessment of odours," Atmospheric Environment, vol. 206, no. JUN., pp. 237-246, 2019.

[17] T. Saravanakumar, N. H. Muoi, and Q. Zhu, "Finite-time sampled-data control of switched stochastic model with nondeterministic actuator faults and saturation nonlinearity," Journal of the Franklin Institute, vol. 357, no. 18, pp. 13637$13665,2020$.

[18] E. Kianmehr, S. Nikkhah, and A. Rabiee, "Multi-objective stochastic model for joint optimal allocation of DG units and network reconfiguration from DG owner's and DisCo's perspectives," Renewable Energy, vol. 132, no. MAR., pp. 471-485, 2019.

[19] G. Kermarrec, I. Neumann, H. Alkhatib, and S. Schon, “The stochastic model for global navigation satellite systems and terrestrial laser scanning observations: a proposal to account for correlations in least squares adjustment," Journal of Applied Geodesy, vol. 13, no. 2, pp. 93-104, 2019.

[20] A. B. Kapranova, "Stochastic model of parallel or sequential processes of deaeration and mixing of granular media using the operation of a centrifugal device as an example," Theoretical Foundations of Chemical Engineering, vol. 53, no. 2, pp. 292-304, 2019.

[21] C. M. Straut and A. Nelson, "Improving chemical security with material control and accountability and inventory management," Journal of Chemical Education, vol. 97, no. 7, pp. 1809-1814, 2020.

[22] J. Zan, Y. Liu, X. Wang, and F. K. Zhang, "Assessment on emergency management capability of chemical industrial parks in Hubei Province," Industrial Safety and Environmental Protection, vol. 46, no. 1, pp. 64-66, 2020.

[23] X. Liu, W. Mao, and G. Yu, "Research on safety deployment optimization technique of fire truck in petrochemical storage tank fire accident," Industrial Safety and Environmental Protection, vol. 45, no. 4, pp. 20-22, 2019.

[24] K. Chen and Z. Deng, "Classification and analysis on the standardized system of occupational safety and health regulation in chemical industry," China Occupational Medicine, vol. 46, no. 2, pp. 239-242, 2019.

[25] Q. Luo, Y. Li, B. Wu, and J. Tong, "Application of microreaction technology in improving safety of fine chemical process," Modern Chemical Industry, vol. 39, no. 4, pp. 63-66, 2019.

[26] A. P. Essoh, F. Monteiro, A. R. Pena, M. Pais, M. Moura, and M. M. Romeiras, "Exploring glucosinolates diversity in Brassicaceae: a genomic and chemical assessment for deciphering abiotic stress tolerance," Plant Physiology and Biochemistry, vol. 150, pp. 151-161, 2020. 